\title{
Publisher Correction to: Effect of glaucoma implant surgery on intraocular pressure reduction, flare count, anterior chamber depth, and corneal endothelium in primary open-angle glaucoma
}

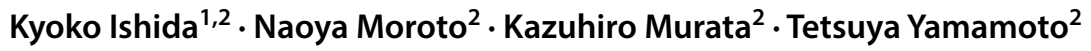

Published online: 12 March 2018

(c) Japanese Ophthalmological Society 2018

\section{Correction to: Jpn J Ophthalmol (2017) 61:334-346 https://doi.org/10.1007/s10384-017-0512-2}

The publisher regrets that the original version of this article unfortunately contained numerous mistakes that occurred during typesetting. Those errors and their corrections are shown below.

\begin{tabular}{lllll}
\hline Page & Column & Line & In the original article & Should be \\
\hline 334 & 1 & 15 & IOP $\geq 5 \mathrm{mmHg}$ & IOP $\geq 5 \mathrm{mmHg}$ \\
336 & 1 & 17 & an IOP $\geq 5 \mathrm{mmHg}$ & an IOP $\geq 5 \mathrm{mmHg}$ \\
& 1 & 19 & an IOP $\geq 5 \mathrm{mmHg}$ & an IOP $\geq 5 \mathrm{mmHg}$ \\
& 1 & 28 & IOP $<5 \mathrm{mmHg}$. & IOP $<5 \mathrm{mmHg}$. \\
& 1 & 42 & the Chi-square & the chi-square \\
& 2 & 7 & patient's & patients' \\
338 & 1 & 1 & IOPs $>18 \mathrm{mmHg}$ & IOPs $>18 \mathrm{mmHg}$ \\
& 1 & 7 & IOPs $>15 \mathrm{mmHg}$ & IOPs $>15 \mathrm{mmHg}$ \\
& 1 & 8 & reduction $>20 \%$ & reduction $>20 \%$ \\
& 2 & 5 & of $<0.3333$ & of $<0.3333$ \\
341 & 1 & 20 & IOP $\geq 5 \mathrm{mmHg}$ & IOP $\geq 5 \mathrm{mmHg}$ \\
& 2 & 18 & IOP $\leq 10 \mathrm{mmHg}$ & IOP $\leq 10 \mathrm{mmHg}$ \\
& 2 & 25 & IOP $\leq 10 \mathrm{mmHg}$ & IOP $\leq 10 \mathrm{mmHg}$ \\
343 & 1 & 8 & $1 \%$ to $52.4 \%$ & 1 to $52.4 \%$ \\
1 & 20 & Chiou et al & Chiou et al. \\
& 2 & 42 & IOP $<5 \mathrm{mmHg}$ & IOP $<5 \mathrm{mmHg}$
\end{tabular}

The original article can be found online at https://doi.org/10.1007/ s10384-017-0512-2.

\begin{tabular}{|c|c|c|c|c|}
\hline Page & Column & Line & In the original article & Should be \\
\hline 344 & 2 & 33 & $\begin{array}{l}\text { Conflicts of inter- } \\
\text { est K. Ishida, N. } \\
\text { Moroto, K. Murata } \\
\text { have no conflict } \\
\text { of interest. T. } \\
\text { Yamamoto, Grants } \\
\text { (Alcon Japan, MSD } \\
\text { Japan, Otsuka, } \\
\text { Pfizer Japan, } \\
\text { Santen, Senju), } \\
\text { Consultant/Advisor } \\
\text { fees (Alcon Japan, } \\
\text { Kowa, Otsuka, } \\
\text { Pfizer Japan, } \\
\text { Rohto, Senju), } \\
\text { Lecture fees (Alcon } \\
\text { Japan, AMO Japan, } \\
\text { CREWT Medical } \\
\text { Systems, John- } \\
\text { son \& Johnson, } \\
\text { Kowa, MSD Japan, } \\
\text { Novartis Pharma, } \\
\text { Otsuka, Pfizer } \\
\text { Japan, R-Tech } \\
\text { Ueno, Santen, } \\
\text { Senju). }\end{array}$ & $\begin{array}{l}\text { Conflicts of interest } \\
\text { K. Ishida, None; N. } \\
\text { Moroto, None; K. } \\
\text { Murata, None; T. } \\
\text { Yamamoto, Grants } \\
\text { (Alcon Japan, MSD } \\
\text { Japan, Otsuka, } \\
\text { Pfizer Japan, Santen, } \\
\text { Senju), Consult- } \\
\text { ant/Advisor fees } \\
\text { (Alcon Japan, } \\
\text { Kowa, Otsuka, } \\
\text { Pfizer Japan, Rohto, } \\
\text { Senju), Lecture fees } \\
\text { (Alcon Japan, AMO } \\
\text { Japan, CREWT } \\
\text { Medical Systems, } \\
\text { Johnson \& Johnson, } \\
\text { Kowa, MSD Japan, } \\
\text { Novartis Pharma, } \\
\text { Otsuka, Pfizer } \\
\text { Japan, R-Tech Ueno, } \\
\text { Santen, Senju). }\end{array}$ \\
\hline
\end{tabular}

Kyoko Ishida

kyoko.ish@gmail.com

1 Department of Ophthalmology, Toho University

Ohashi Medical Center, 2-17-6 Ohashi, Meguro-ku,

Tokyo 153-8515, Japan

2 Department of Ophthalmology, Gifu University Graduate School of Medicine, Gifu, Japan 